Cite this: Soft Matter, 2014, 10, 5212

Received 21st March 2014 Accepted 17th April 2014 DOI: $10.1039 / \mathrm{c} 4$ sm00625a www.rsc.org/softmatter

\section{Surfactant or block copolymer micelles? Structural properties of a series of well-defined $n$-alkyl-PEO micelles in water studied by SANS}

\author{
Thomas Zinn, $\dagger^{\mathrm{a}}$ Lutz Willner, ${ }^{a}$ Reidar Lund, ${ }^{\star b}$ Vitaliy Pipich, ${ }^{c}$ Marie-Sousai Appavou ${ }^{c}$ \\ and Dieter Richter ${ }^{a}$
}

\begin{abstract}
Here we present an extensive small-angle neutron scattering (SANS) structural characterization of micelles formed by poly(ethylene oxide)-mono- $n$-alkyl ethers $\left(C_{n}-P E O x\right)$ in dilute aqueous solution. Chemically, $\mathrm{C}_{n}$-PEOx can be considered as a hybrid between a low-molecular weight surfactant and an amphiphilic block copolymer. The present system, prepared through anionic polymerization techniques, is better defined than other commercially available polymers and allows a very precise and systematic testing of the theoretical predictions from thermodynamical models. The equilibrium micellar properties were elaborated by systematically varying the $n$-alkyl chain length $(n)$ at constant PEO molecular weight or increasing the soluble block size $(x)$, respectively. The structure was reminiscent of typical spherical starlike micelles i.e. a constant core density profile, $\sim r^{0}$, and a diffuse corona density profile, $\sim r^{-4 / 3}$. Through a careful quantitative analysis of the scattering data, it is found that the aggregation number, $N_{\text {agg }}$ initially rapidly decreases with increasing PEO length until it becomes independent at higher PEO molecular weight as expected for star-like micelles. On the other hand, the dependency on the $n$-alkyl length is significantly stronger than that expected from the theories for star-like block copolymer micelles, $N_{\text {agg }} \sim$ $n^{2}$ similar to what is expected for surfactant micelles. Hence the observed aggregation behavior suggests that the $C_{n}-P E O x$ micelles exhibit a behavior that can be considered as a hybrid between low-molecular weight surfactant micelles and diblock copolymer micelles.
\end{abstract}

\section{Introduction}

The self-assembly of block copolymers in micellar structures has been widely studied in theoretical studies, ${ }^{1-9,26}$ experiments ${ }^{10-17}$ and computer simulations. ${ }^{18-20}$ A general overview on this topic can be found in several review articles and books. ${ }^{21-25}$

Diblock copolymers and small surfactant molecules basically show the same spontaneous self-association of single molecules (unimers) into micellar aggregates. Depending on the conditions and molecular parameters, spherical, cylindrical or vesicular micelles are usually formed. ${ }^{22}$ Nagarajan and Ganesh ${ }^{5}$ developed a thermodynamic treatment of block copolymers in a selective solvent by deriving the total Gibbs free energy for a micellar solution. Analytical and self-consistent calculations are inherently difficult for such multicomponent systems. However,

aülich Centre for Neutron Science JCNS and Institute for Complex Systems ICS, Forschungszentrum Jülich GmbH, 52425 Jülich, Germany. E-mail: l.willner@ fz-juelich.de

${ }^{b}$ Department of Chemistry, University of Oslo, Postboks 1033 Blindern, 0315 Oslo, Norway.E-mail: reidar.lund@kjemi.uio.no

'Jülich Centre for Neutron Science JCNS, Forschungszentrum Jülich GmbH, Outstation at MLZ, Lichtenbergstraße 1, 85747 Garching, Germany

$\dagger$ Present address: Department of Chemistry, University of Oslo, Postboks 1033 Blindern, 0315 Oslo, Norway. for block copolymer micelles it has been shown that the pseudophase approximation ${ }^{5}$ i.e. that the micelles can be viewed thermodynamically as a distinct "phase", is a reasonable assumption. This is valid for a very low cmc (critical micelle concentration), i.e. when the fraction of free chains is comparatively small and the aggregation number is large. This assumption is usually fulfilled for amphiphilic block copolymers in water where the interfacial tension assumes large values. Moreover by utilizing the self-similar properties of polymers, scaling theories have been applied with great success..$^{3,4,89}$ These theories provide rather simple predictions of the general dependency of molecular parameters that can be systematically tested by experiments. For low-molecular weight surfactants, however, both the pseudo-phase approximation and scaling theories cannot generally be used and demands much more detailed analysis. It is therefore interesting to study systems which are hybrids between the two, i.e. amphiphilic molecules where one part is polymer-like and the other is of low molecular weight. In this way the limitations of the theories can be elaborated.

Small-angle X-ray/neutron scattering (SAXS/SANS) techniques have proven to be a powerful method in order to examine the structures of micellar aggregates on a nanometer length scale and many comparisons between experimental data 
and theories have been reported in the literature..$^{\mathbf{1 3 2 7 - 2 9}}$ Contrary to many other techniques, SAXS/SANS provide quantitative information of the detailed shape and size of nanostructures in solution. In addition, SANS provides additional advantages in terms of contrast variation through relatively simple hydrogen/ deuterium substitution that allows the different parts of the micelles (core, shell) to be selectively highlighted. However, there are only very few systematic studies testing the existing thermodynamical theories for micelles by varying molecular parameters and investigating the resulting structures using SANS/SAXS. Amphiphilic block copolymers of the type poly(diene)-PEO, with polyisoprene or polybutadiene, or the saturated analogues PE or PEP, as insoluble blocks and $n$-alkyl PEOs have been studied more intensively in the past because of their chemical similarities to low-molecular weight non-ionic surfactants, $\mathrm{C}_{n} \mathrm{E}_{m}$. For instance, commercially available "Brij" surfactants, $\mathrm{C}_{n}-\mathrm{PEO},{ }^{\mathbf{3 0 , 3 1}}$ poly(ethylene-co-propylene)- $b$-poly(ethylene oxide), PEP- $b$ PEO, ${ }^{32-35}$ poly(butadiene)- $b$-poly(ethylene oxide) $\mathrm{PB}-b$ - $\mathrm{PEO}^{36-38}$ or poly(styrene)- $b$-poly(ethylene oxide), PS- $b$-PEO ${ }^{39,40}$ have been used to study the structure of block copolymer micelles by scattering techniques applying X-rays or neutrons. $\mathrm{C}_{n}-\mathrm{PEO}$ surfactants are interesting because they can be purchased in various PEO lengths and thereby bridge the gap between low-molecular weight surfactants and polymeric surfactants. However, as Brij surfactants are industrial products, impurities might exist which requires care when comparing theory and experiments.

In this paper, we present a structural investigation of poly(ethylene oxide)-mono- $n$-alkyl ether block copolymer micelles by SANS. These $\mathrm{C}_{n}-\mathrm{PEO}$ polymers were synthesized using stateof-the-art anionic polymerization leading to very well defined materials in terms of very low degree of impurities and near monodisperse PEO blocks $\left(M_{\mathrm{w}} / M_{\mathrm{n}} \leq 1.05\right)$. Moreover, the control of the synthesis allowed us to accurately vary both the length of the $n$-alkyl group and the PEO block beyond that for Brij surfactants which are generally only available with $\mathrm{C}_{12}$ or $\mathrm{C}_{18}$ hydrophobic blocks and rather short PEO segments. By a careful quantitative analysis of the SANS data with a core-shell model, we analysed the micellar structure for a series of $\mathrm{C}_{n}$ PEO5 polymers with $n$ ranging from 18 to 30 and for $\mathrm{C}_{27}$ with three further PEO molecular weights of $10 \mathrm{~kg} \mathrm{~mol}^{-1}, 20 \mathrm{~kg}$ $\mathrm{mol}^{-1}$ and $40 \mathrm{~kg} \mathrm{~mol}{ }^{-1}$. In addition from previous work we know that the system is able to attain equilibrium since molecular exchange is active for all $n$-alkyl-PEO micelles. ${ }^{41}$ This allows an accurate and sensible comparison with existing thermodynamic predictions which often is complicated for regular amphiphilic block copolymers due to slow equilibration kinetics and non-ergodic behavior. ${ }^{42}$

\section{Theoretical background}

In analogy to the micellization of low-molecular weight surfactants A-B block copolymers spontaneously self-assemble when dispersed in a selective solvent. If the solvent is selective for the A block, microdomains of a collapsed B block surrounded by a swollen A block are formed. The individual polymer blocks are characterized by the degree of polymerization $N_{\mathrm{A}(\mathrm{B})}$, a statistical segment length $l_{\mathrm{A}(\mathrm{B})}$ and monomer volume $v_{\mathrm{A}(\mathrm{B})} \sim l_{\mathrm{A}(\mathrm{B})}{ }^{3}$.
The structure of the microdomains primarily depends on the degree of polymerization $N=N_{\mathrm{A}}+N_{\mathrm{B}}$, the composition and the interactions between the constituents that are thermodynamically described by the Flory-Huggins interaction parameter $\chi$. With the assumption of a highly asymmetric linear diblock $\left(N_{\mathrm{A}} \gg N_{\mathrm{B}}\right)$ and the dilute solution limit micellar aggregates typically reveal a spherical shape. The topology of these micelles is divided into two distinct regions: the micellar core with a radius $R_{\mathrm{c}}$ and the micellar corona with a thickness $D=R_{\mathrm{m}}-R_{\mathrm{c}}$ where $R_{\mathrm{m}}$ is the overall micellar radius. The structure of the micellar entity on a thermodynamic level is given by the free energy per aggregated chain in the assembly $\mathscr{F}_{\text {mic }}$ which can be expressed in terms of mentioned parameters of the polymer. $\mathscr{F}$ mic can then be summarized by three main essential contributions:

$$
\mathscr{\mathscr { F }}_{\text {mic }}=\tilde{\mathscr{F}}_{\text {core }}+\mathscr{\mathscr { F }}_{\text {corona }}+\mathscr{\mathscr { F }}_{\text {int }}
$$

where $\mathscr{F}_{\text {core }}$ and $\mathscr{F}_{\text {corona }}$ contain entropic terms that describe the stretching of the core blocks and osmotic crowding of the corona blocks, respectively. The last term $\mathscr{F}_{\text {int }}$ is the interfacial free energy associated with the creation of an interface separating the core region from the corona. This enthalpic contribution favors micellization and is given by the interfacial area $A_{\text {int }}$ and the interfacial tension $\gamma$. The Flory-Huggins theory relates $\chi$ via the Helfand-equation to $\gamma: \gamma \sim \chi^{1 / 2}$. Within a selfconsistent mean-field theory the microdomain formation is distinguished to three distinct regimes: the weak (WSL), ${ }^{43}$ the strong (SSL) ${ }^{\mathbf{4}}$ and the super strong segregation limit (SSSL). ${ }^{\mathbf{8 , 4 5}}$ In the WSL the polymer is only weakly perturbed from a Gaussian coil behavior $(\chi N \geq 10$ ) whereas for sufficiently large polymers and a high enough interfacial tension a sharp interface separates the A and B domains from each other $(\chi N \gg 10)$. Furthermore, the microdomain size (e.g. the core radius $R_{\mathrm{c}}$ ) is controlled by the number of diblock copolymers $\left(N_{\text {agg }}\right)$ in the structure. Applying a simple geometric space filling argument the scaling behavior is given by

$$
R_{\mathrm{c}}=\sqrt[3]{\frac{3 N_{\mathrm{agg}} V_{\mathrm{B}}}{4 \pi N_{\mathrm{Avo}}}} \simeq N_{\mathrm{agg}}{ }^{1 / 3} N_{\mathrm{B}}{ }^{1 / 3} l_{\mathrm{B}}
$$

where $N_{\text {Avo }}$ is Avogadro's number and $V_{\mathrm{B}}$ the molecular volume of the B block. At this point the growth of the microdomain is limited by two geometrical constrains: (i) the radius of the domain cannot be larger than the contour length of the polymer ( $L_{\mathrm{B}}=N_{\mathrm{B}} l_{\mathrm{B}}$ ) and (ii) in thermodynamic equilibrium the surface is maximally occupied by A-B junctions yielding a certain value of $N_{\text {agg. }}$. If either (i) or (ii) is reached this is qualitatively described by the SSSL. The SSSL differs from the SSL in a way that the interactions between A and B are super-strong meaning that the interfacial energy dominates over the contribution of the corona, $\mathscr{F}$ corona. As a consequence in this case a nearly fully elongated conformation of the B-block is assumed.

The micelle free energy per aggregated chain in units of $k_{\mathrm{B}} T$ is given by eqn (3).

$$
\mathscr{F}_{\text {mic }} \simeq N_{\mathrm{agg}}{ }^{2 / 3} N_{\mathrm{B}}{ }^{-1 / 3}+\frac{N_{\mathrm{agg}}{ }^{1 / 2}}{\sqrt{2 \pi}} \ln \frac{D}{R_{\mathrm{c}}}+4 \pi \gamma N_{\mathrm{agg}}{ }^{-1 / 3} N_{\mathrm{B}}{ }^{2 / 3}
$$


The equilibrium aggregation number of the micellar entity is given by minimization of the free energy with respect to $N_{\mathrm{agg}}$. This leads to the following scaling expressions

$$
N_{\mathrm{agg}} \sim \gamma^{6 / 5} N_{\mathrm{B}}^{4 / 5}\left[\ln \left(\frac{D}{R_{\mathrm{c}}}\right)\right]^{-6 / 5}
$$

and the equilibrium micellar radius is derived to

$$
R_{\mathrm{m}} \sim \gamma^{6 / 25} N_{\mathrm{B}}{ }^{4 / 25} N_{\mathrm{A}}{ }^{3 / 5}\left[\ln \left(\frac{D}{R_{\mathrm{c}}}\right)\right]^{-6 / 25}
$$

The minimization of eqn (3) leads to a term $\left(N_{\mathrm{B}}{ }^{-2} N_{\mathrm{agg}}\right)^{1 / 6}$ which can be approximated in the limit of $N_{\mathrm{A}} \gg N_{\mathrm{B}}$ by $1 .^{3}$ The core radius is obtained by inserting eqn (4) into (2) which leads to the following expression:

$$
R_{\mathrm{c}} \sim \gamma^{2 / 5} N_{\mathrm{B}}^{3 / 5}\left[\ln \left(\frac{D}{R_{\mathrm{c}}}\right)\right]^{-2 / 5}
$$

Eqn (4)-(6) are obtained for the SSL but as the $\gamma$ increases the transition to the SSSL occurs at a critical value $\gamma^{*}$ where the core block chains exhibit an almost fully elongated conformation:

$$
\gamma^{*} \sim N_{\mathrm{B}} \ln \left(\frac{D}{R_{\mathrm{c}}}\right)
$$

The latter eqn (7) is obtained from eqn (6) by assuming $R_{\mathrm{c}} \sim N_{\mathrm{B}}$. Inserting eqn (7) into eqn (4) yields a characteristic $N_{\mathrm{B}}{ }^{2}$ dependence on the aggregation number:

$$
N_{\text {agg }} \sim N_{\mathrm{B}}^{2}
$$

This type of behavior is typically observed for low-molecular weight surfactant micelles. Contrary to amphiphilic block copolymer micelles the $N_{\mathrm{B}}{ }^{2}$-dependence is satisfied inherently. In this case the micellar size and shape are given by geometrical constrains due to the chain packing inside the core and was introduced by Israelachvili and coworkers more than 30 years ago. ${ }^{46}$ In order to emphasize the difference to the surfactant approach the interfacial tension controls the morphology of the micelle rather than a balance between the two opposing forces of the chain deformation and minimization of the interfacial area.

\section{Experimental section}

\subsection{Materials and solutions}

Synthesis and characterization. The poly(ethylene oxide) mono- $n$-alkyl ethers, $\mathrm{C}_{n}-\mathrm{PEO} x$ (with $x=5,10,20,40 \mathrm{~kg} \mathrm{~mol}^{-1}$ ) were synthesized by living anionic ring opening polymerization of ethylene oxide using an 80:20 mixture of an 1-alcohol $\left(\mathrm{C}_{n} \mathrm{H}_{2 n+1} \mathrm{OH}\right.$ with $\left.n=18,21,24,27,28,30\right)$ (Aldrich) with its corresponding potassium-1-alkoxide $\left(\mathrm{C}_{n} \mathrm{H}_{2 n+1} \mathrm{O}^{-} \mathrm{K}^{+}\right)$as the initiating system. Deuterated polymers were prepared accordingly by polymerizing perdeuterated ethylene oxide, EO- $\mathrm{d}_{4}$ (Eurisotop; 98\%D). For polymer $\mathrm{C}_{18}-\mathrm{PEO} 5$ additionally deuterated 1-octadecanol- $\mathrm{d}_{37}$ (Eurisotop, 98\%D) was used whereas for all other polymers the 1-alkohols were always proteated. The polymerizations were carried out under high vacuum in toluene at $95{ }^{\circ} \mathrm{C}$. At this temperature the potassium-1-alkoxides are soluble and the $\mathrm{H}^{+} / \mathrm{K}^{+}$exchange is fast providing homogeneous initiation/propagation conditions. Generally, working with the ethylene oxide monomer requires strong safety precautions because of the high toxicity and inflammability of this material. Moreover, EO is a liquid gas $\left(\mathrm{bp}=10^{\circ} \mathrm{C}\right)$ evolving overpressure in particular in the beginning of the polymerization reaction where not yet much of the EO monomer has been polymerized. Therefore, pressure tested (12 bars) heavy walled Schlenk bombs fitted with Teflon plug valves were taken as polymerization reactors. After $24 \mathrm{~h}$ the overpressure had entirely disappeared indicating complete conversion of the monomer. The living polymers were terminated by adding acetic acid leading to a hydroxy group at the terminal position. The polymers were precipitated twice in cold acetone at $-20{ }^{\circ} \mathrm{C}$ and isolated by centrifugation. Finally, they were dissolved in benzene, filtered and freeze-dried in high vacuum.

The resulting polymers were characterized by a combination of size exclusion chromatography (SEC) and ${ }^{1} \mathrm{H}-\mathrm{NMR}$ (proteated polymers). NMR-spectra were recorded in $\mathrm{CDCl}_{3}$. The number average molecular weight of the PEO component was then calculated using the integral intensity of the $n$-alkyl block as the internal reference. SEC measurements were done with tetrahydrofuran $/ N, N$-dimethylacetamide $(85 / 15)$ as the eluent at $50{ }^{\circ} \mathrm{C}$ using a set of three Agilent PlusPore GPC columns with a continuous distribution of pore sizes and PEO standards for calibration. Determined polydispersity indices were typically small in the order of $M_{\mathrm{w}} / M_{\mathrm{n}} \leq 1.04$. The chromatograms, however, revealed small contents (1\%) of an impurity at elution volumes corresponding to approximately twice the alkyl-PEO molecular weight. Most likely this is due to the presence of spurious amounts of water which cannot be removed even with rigorous drying procedures for the monomer and solvent. Water can participate in the $\mathrm{H}^{+} / \mathrm{K}^{+}$exchange described above and thus may act as a difunctional initiator for the EO polymerization. Deuterated polymers were only analyzed by SEC. Their number average molecular weights were calculated by relating the SEC data to those of the proteated counterparts and by taking into account the deuteration. The important polymer characteristics are summarized in Table 1.

Scattering length densities. The scattering length densities of solvents and polymers were calculated according to $\rho=\left(N_{\mathrm{Avo}} d / M\right) \sum_{i} b_{i}$ here $b_{i}$ denotes the coherent scattering length of an individual atom in the $n$-alkyl block, the EO repeat unit or the water molecules. $M$ is the respective molecular weight and $N_{\text {Avo }}=6.022 \times 10^{23}$ Avogadro's number. For the calculation of $\rho$ of the hydrophobic block the chemical composition of the $n$-alkyl group $\left(\mathrm{C}_{n} \mathrm{H}_{2 n+1}\right)$ was taken because this part was considered to be fully segregated forming the core of the micelle. Mass densities were those of the $n$-alkanes as tabulated in Landolt-Börnstein. ${ }^{47}$ The calculated scattering length densities for the different $n$-alkyls are summarized in Table 2. In the case of PEO the solution densities were taken as determined by Sommer et al.: ${ }^{31} d(\mathrm{~h}-\mathrm{PEO})=1.196 \mathrm{~g} \mathrm{~cm}^{-3}$ and 
Table 1 Molecular characteristics of $C_{n}-$ PEOx polymers

\begin{tabular}{llll}
\hline Polymer & Label $^{a}$ & $M_{\mathrm{C}_{n}}{ }^{b}$ & $M_{\mathrm{n}}{ }^{c}$ (PEO) \\
\hline $\mathrm{C}_{18}$-PEO5 & hh & 254.5 & 4.0 \\
& dd & 292.2 & 4.0 \\
$\mathrm{C}_{21}$-PEO5 & hh & 296.6 & 4.1 \\
& hd & & 4.4 \\
$\mathrm{C}_{24}$-PEO5 & hh & 338.7 & 4.2 \\
& hd & & 4.2 \\
$\mathrm{C}_{27}$-PEO5 & hh & 380.7 & 4.2 \\
$\mathrm{C}_{27}$-PEO10 & hd & & 4.4 \\
& hh & & 10.4 \\
$\mathrm{C}_{27}$-PEO20 & hd & & 10.9 \\
& hh & & 21.2 \\
$\mathrm{C}_{27}$-PEO40 & hd & & 20.4 \\
& hh & & 36.0 \\
$\mathrm{C}_{28}$-PEO5 & hd & & 38.5 \\
& hh & 394.8 & 4.6 \\
$\mathrm{C}_{30}$-PEO5 & hd & & 4.8 \\
& hh & 422.8 & 4.3 \\
& hd & & 4.1
\end{tabular}

${ }^{a}$ Type of isotope labelling: (h) hydrogen/(d) deuterium. ${ }^{b}$ Molecular weight of respective $n$-alkane $\left[\mathrm{g} \mathrm{mol}^{-1}\right] .{ }^{c} M_{\mathrm{n}}$ (PEO) in $\left[\mathrm{kg} \mathrm{mol}^{-1}\right]$.

Table 2 Scattering length density of $n$-alkyl block, $\rho_{\mathrm{C}_{n^{\prime}}}$ in $10^{10} \mathrm{~cm}^{-2}$

\begin{tabular}{lrlllll}
\hline Label & $\mathrm{C}_{18}$ & $\mathrm{C}_{21}$ & $\mathrm{C}_{24}$ & $\mathrm{C}_{27}$ & $\mathrm{C}_{28}$ & $\mathrm{C}_{20}$ \\
\hline $\mathrm{h}$ & -0.349 & -0.343 & -0.339 & -0.335 & -0.334 & -0.333 \\
$\mathrm{~d}$ & 6.520 & & & & &
\end{tabular}

$d(\mathrm{~d}-\mathrm{PEO})=1.305 \mathrm{~g} \mathrm{~cm}^{-3}$. The corresponding scattering length densities were then calculated to be $\rho_{\mathrm{h}-\mathrm{EO}}=0.676 \times 10^{10} \mathrm{~cm}^{-2}$ and $\rho_{\mathrm{d}-\mathrm{EO}}=7.421 \times 10^{10} \mathrm{~cm}^{-2}$, respectively. Scattering length densities of the pure solvents $\mathrm{H}_{2} \mathrm{O}$ and $\mathrm{D}_{2} \mathrm{O}$ are $\rho_{\mathrm{H}_{2} \mathrm{O}}=-5.599 \times$ $10^{9} \mathrm{~cm}^{-2}$ and $\rho_{\mathrm{D}_{2} \mathrm{O}}=6.357 \times 10^{10} \mathrm{~cm}^{-2}$. For polymers $\mathrm{C}_{27}-\mathrm{PEO} x$ we have used a $\mathrm{H}_{2} \mathrm{O}-\mathrm{D}_{2} \mathrm{O}$ water mixture $\left(\phi_{\mathrm{D}_{2} \mathrm{O}}=0.56\right)$ as the solvent having an intermediate contrast of $\rho_{\mathrm{H}_{2} \mathrm{O} / \mathrm{D}_{2} \mathrm{O}}=3.360 \times$ $10^{10} \mathrm{~cm}^{-2}$. Densities at $20{ }^{\circ} \mathrm{C}$ were taken for all calculations. Temperature effects were proven to be negligible in the studied temperature range.

Sample preparation. Micellar solutions were obtained according to the following general mixing protocol. At first a stock solution of $\phi=1 \%$ polymer volume fraction was prepared by weighing in the pure components. Calculation of volume fractions was done assuming additivity of volumes. To ensure complete dissolution and micellar equilibration the samples were heated up to $60{ }^{\circ} \mathrm{C}$ for approximately 3 hours. We should note that this procedure lead to equilibrium structures as we know from kinetic studies on these materials. ${ }^{41}$ After that solutions were slowly cooled down to room-temperature overnight while shaking. The stock solutions were diluted without heating again to $\phi=0.25 \%, \mathrm{C}_{27}-\mathrm{PEO} 40$ was diluted to $\phi=0.1 \%$. SANS measurements were carried out in standard Hellma Quartz cells with $1 \mathrm{~mm}\left(\mathrm{H}_{2} \mathrm{O}\right.$ solutions $)$ and $2 \mathrm{~mm}\left(\mathrm{D}_{2} \mathrm{O}\right.$ solutions and $\mathrm{H}_{2} \mathrm{O} / \mathrm{D}_{2} \mathrm{O}$ solutions) path lengths.

\subsection{SANS and data evaluation}

The small-angle neutron scattering (SANS) experiments were conducted at KWS-1 and KWS-2 instruments located at HeinzMeier-Leibnitz Zentrum (MLZ) in Garching, Germany. Measurements of $\mathrm{C}_{n}-\mathrm{PEO} 5$ were carried out under full contrast, i.e. hh in $\mathrm{D}_{2} \mathrm{O}$ and hd/dd in $\mathrm{H}_{2} \mathrm{O}$. The $\mathrm{C}_{27}-\mathrm{PEO} x$ samples were measured in the isotopic water mixture $\left(\phi_{\mathrm{D}_{2} \mathrm{O}}=0.56\right)$ providing the intermediate contrast. The latter contrast condition was chosen in order to study micellar exchange kinetics in these systems. However, a discussion of the kinetics goes beyond the scope of this work. Sample-to-detector distances were 2 and $8 \mathrm{~m}$ and $20 \mathrm{~m}$ additionally for $\mathrm{C}_{27}-\mathrm{PEO} x$ with $x>10$. Collimation lengths were set identical to sample-to-detector distances, except for $2 \mathrm{~m}$ where an $8 \mathrm{~m}$ collimation length was chosen in order to avoid detector saturation. KWS measurements were carried out with a neutron wavelength of $\lambda=7 \AA$ and a wavelength spread $\frac{\Delta \lambda}{\lambda}=10 \%$ at KWS-1 and $\frac{\Delta \lambda}{\lambda}=20 \%$ at KWS-2, respectively. The 2 and $8 \mathrm{~m}$ settings yield a $Q$-range of $0.006 \leq Q$ $\left[\AA^{-1}\right] \leq 0.2$ and for the $20 \mathrm{~m}$ setup we could extend the range down to $Q=0.003 \AA^{-1}$, where $Q=4 \pi / \lambda \sin \theta$ is the momentum transfer and $2 \theta$ is the scattering angle. Scattered intensities were corrected for detector sensitivity, empty cell scattering, electronic noise and dead time effects due to detector electronics. The scattering data were set on absolute scale using plexiglass as a calibrated secondary standard. The thus obtained absolute normalized differential scattering cross-section $\mathrm{d} \Sigma / \mathrm{d} \Omega(Q)$ in $\mathrm{cm}^{-1}$ was further corrected for incoherent scattering contributions. Incoherent scattering of the solvent was measured separately, whereas the incoherent scattering of the polymer was calculated. All data reductions were done by using the computer software QtiKWS available at MLZ in Garching.48

The coherent macroscopic scattering cross-section of the micellar solutions $\mathrm{d} \Sigma / \mathrm{d} \Omega(Q)$ in the dilute limit i.e. $S(Q) \approx 1$ was analyzed according to

$$
\frac{\mathrm{d} \Sigma}{\mathrm{d} \Omega}(Q) \approx \frac{\phi}{N_{\mathrm{agg}}\left(V_{\mathrm{C}_{n}}+V_{\mathrm{PEO}}\right)} P(Q)
$$

with $\phi$ the polymer volume fraction, $N_{\text {agg }}$ the aggregation number and $V_{\mathrm{C}_{n}}+V_{\mathrm{PEO}}$ the total molar volume of the block copolymer. $P(Q)$ relies on a form factor model established for spherical star-like block copolymer micelles by Pedersen and co-workers. ${ }^{28}$ This model includes all important structural parameters of a micelle i.e. the aggregation number, $N_{\text {agg }}$ which determines the micellar core radius $R_{\mathrm{c}}$ (see eqn (2)) and the overall micellar radius, $R_{\mathrm{m}}$.

The micellar form factor $P(Q)$ is given by

$$
\begin{aligned}
P(Q) & =\left(\rho_{\mathrm{C}_{n}}-\rho_{0}\right)^{2} N_{\mathrm{agg}}{ }^{2} V_{\mathrm{C}_{n}}{ }^{2} A_{\mathrm{c}}{ }^{2}(Q)+\left(\rho_{\mathrm{PEO}}-\rho_{0}\right)^{2} N_{\mathrm{agg}} \\
& \left(N_{\mathrm{agg}}-B(0)\right) V_{\mathrm{PEO}} A_{\mathrm{sh}}{ }^{2}(Q)+2\left(\rho_{\mathrm{C}_{n}}-\rho_{0}\right)\left(\rho_{\mathrm{PEO}}-\rho_{0}\right) \\
& N_{\mathrm{agg}}{ }^{2} V_{\mathrm{C}_{n}} V_{\mathrm{PEO}} A_{\mathrm{c}}(Q) A_{\mathrm{sh}}(Q)+V_{\mathrm{PEO}} \Delta \rho_{\mathrm{sh}}{ }^{2} B(Q)
\end{aligned}
$$

where $\Delta \rho_{\mathrm{c}, \mathrm{sh}}=\rho_{\mathrm{c}, \mathrm{sh}}-\rho_{0}$ with $\rho_{0}$ the scattering length density of $\mathrm{H}_{2} \mathrm{O}, \mathrm{D}_{2} \mathrm{O}$ or $\mathrm{H}_{2} \mathrm{O} / \mathrm{D}_{2} \mathrm{O}$, and the scattering amplitudes $A_{\mathrm{c}}(Q) / A_{\mathrm{sh}}(Q)$ for the core and shell region, respectively. $B(Q)$ describes an effective scattering from the internal structure of the coronal polymer chains ("blob-scattering") ${ }^{23,49}$ and is added incoherently:50 


$$
B(Q)=\frac{P_{\text {Beau }}(Q)}{1+\nu P_{\text {Beau }}(Q)}
$$

$\nu$ is a parameter describing the effective chain-chain correlations inside the shell domain and thus $\nu$ represents an apparent concentration inside the corona. The form factor of interacting chains $P_{\text {Beau }}(Q)$ in the shell region can be modeled by using an empiric approach for the characteristic length scale of a selfavoiding chain introduced by Beaucage: ${ }^{51}$

$$
P_{\text {Beau }}(Q)=\mathrm{e}^{-Q^{2} R_{g}{ }^{2} / 3}+\frac{d_{\mathrm{f}}}{R_{g} d_{\mathrm{f}}} \Gamma\left(\frac{d_{\mathrm{f}}}{2}\right)\left(\frac{\operatorname{erf}\left(\frac{k Q R_{g}}{\sqrt{6}}\right)}{Q}\right)^{d_{\mathrm{f}}}
$$

where $R_{g}$ is radius of gyration for PEO, $d_{\mathrm{f}}=1.7$ is the fractal dimension for a polymer in a good solvent and $k$ is a empirical constant set to $1.06 . .^{51}$

The individual $A_{\mathrm{c}}(Q)$ for the core and the shell $A_{\mathrm{sh}}(Q)$ are calculated based on assuming a compact homogeneous core density profile, $n_{\text {core }}=$ const. and the star-like shell density profile, $n_{\text {shell }} \sim r^{-4 / 3}$, respectively. ${ }^{52}$ Thus, the scattering amplitudes can be written as:

$$
\begin{gathered}
A_{\mathrm{c}}(Q)=\frac{3\left(\sin \left(Q R_{\mathrm{c}}\right)-Q R_{\mathrm{c}} \cos \left(Q R_{\mathrm{c}}\right)\right)}{\left(Q R_{\mathrm{c}}\right)^{3}} \times \mathrm{e}^{-Q^{2} \sigma_{\text {int }}{ }^{2} / 2} \\
A_{\mathrm{sh}}(Q)=\frac{1}{C} \int_{R_{\mathrm{c}}}^{\infty} \mathrm{d} r \frac{4 \pi r^{2} r^{-4 / 3}}{1+\mathrm{e}^{\left(r-R_{\mathrm{m}}\right) / \sigma_{\mathrm{m}} R_{\mathrm{m}}}} \frac{\sin (Q r)}{Q r} \times \mathrm{e}^{-Q^{2} \sigma_{\mathrm{int}}{ }^{2} / 2}
\end{gathered}
$$

where $C$ is a normalization constant
$\left.C=\int_{R_{c}}^{\infty} \frac{4 \pi r^{2} r^{-4 / 3}}{1+\mathrm{e}^{\left(r-R_{m}\right) / \sigma_{m} R_{m}}} \mathrm{~d} r\right)$. In eqn (13) and (14) the Gaussian factor gives a smooth core-corona interface where $\sigma_{\text {int }}$ is a measure of the surface roughness. The Fermi cut-off function in eqn (14) is used to terminate the corona region to finite size. For the analysis $\sigma_{\mathrm{m}}$ was set to $10 \%$ of $R_{\mathrm{m}}$. Finally, data analysis incorporates instrumental resolution effects according to a wavelength spread, finite collimation and detector resolution. ${ }^{53,54}$

\section{Results and discussion}

The macroscopic scattering cross-sections, $\mathrm{d} \Sigma / \mathrm{d} \Omega(Q)$, for $\mathrm{C}_{n}$-PEOx micelles in dilute solution $(\phi=0.25)$ are shown in Fig. 1(a) and (b) in a double logarithmic representation. The scattered intensity is normalized by $C=N_{\mathrm{Avo}}\left(V_{\mathrm{C}_{n}}+V_{\mathrm{PEO}}\right) /$ $\left(\phi\left(\Delta \rho_{\mathrm{c}} V_{\mathrm{C}_{n}}+\Delta \rho_{\mathrm{sh}} V_{\mathrm{PEO}}\right)^{2}\right)$ considering concentration, contrast and volumes thus directly yielding $N_{\text {agg }}$ at low $Q$. For better visibility the scattering curves in Fig. 1(b) have been shifted by different factors. The scattering pattern of the micellar solutions exhibit characteristic features like a low $Q$ Guinier-plateau and at high $Q$ a power law dependence of $Q^{-1.7}$ reflecting the semi-dilute nature of the polymer shell domain. ${ }^{33}$ In between the scattered intensity shows a steep decay in a rather narrow $Q$ window. We note that due to the low intensity very close to the incoherent background the apparent slope at high $Q$ sensitively depends on the data reduction. In particular, at low polymer concentration the data statistics is generally poor and the exponent cannot be determined with high precision. However, the necessary structural micellar properties $N_{\mathrm{agg}}$ and $R_{\mathrm{m}}$ were rather unaffected by these variations in the high $Q$ region. Detailed structural parameters were obtained by fitting the data with the spherical core-shell model applying least-square fit routines. Best-fit curves are shown as solid lines in Fig. 1. In order to reduce the number of free parameters the block molecular volumes $V_{n}$ and $V_{\text {PEO }}$, the polymer volume fractions $\phi$ and the scattering length densities $\rho$ were taken as obtained from the polymer characterization or calculated as described in the experimental section. These values were hold fixed throughout the whole analysis. $\nu$ was found to best describe the data by a value close to 0.2 and was kept constant at this value. Any possible structure factor contributions were considered to be negligible at $0.25 \%$ polymer volume fraction. In order to confirm this we have accounted for deviations from the dilute regime by means of a second virial coefficient, $A_{2}$. As an example the second virial coefficient for $\mathrm{C}_{24}$-PEO5 micelles, $A_{2} \approx 1.0 \times 10^{-4} \mathrm{~cm}^{3} \mathrm{~mol} \mathrm{~g}^{-2}$, was deduced from three different concentrations, $\phi=0.25 \%$, $0.5 \%$ and $1 \%$ (data not shown). From trial fits we could confirm
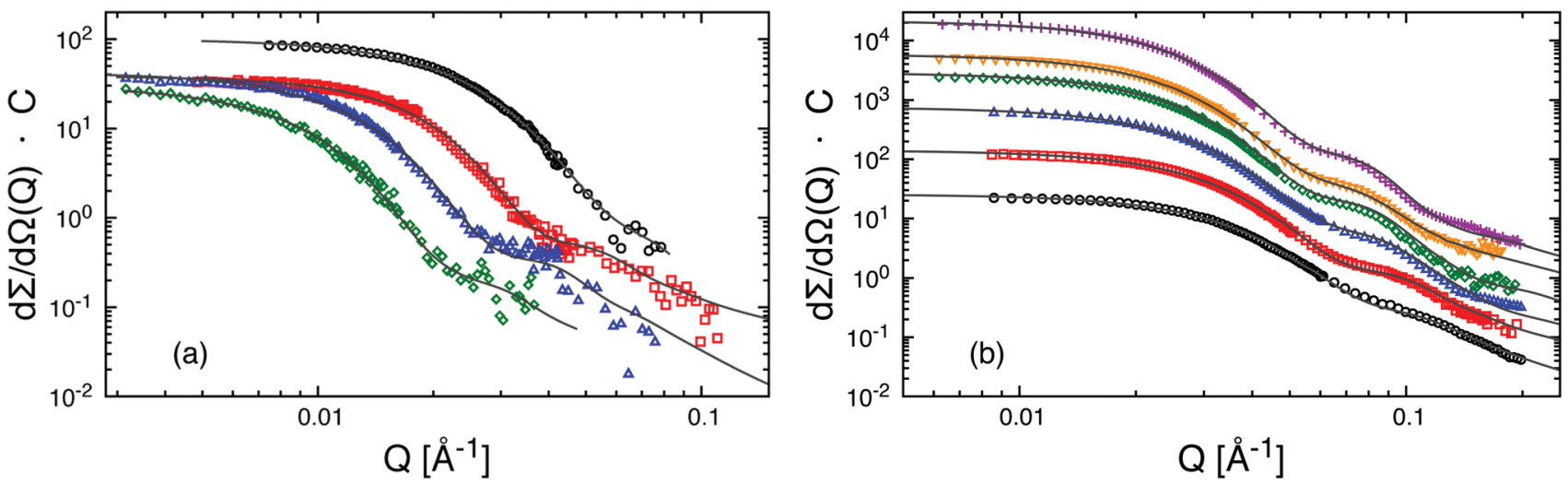

Fig. 1 Form factors of fully proteated $\mathrm{C}_{n}-\mathrm{PEO} x$ (hh) spherical micelles in dilute aqueous solution at $25^{\circ} \mathrm{C}:(\mathrm{a}) \mathrm{C}_{27}-\mathrm{PEO} x, x=5,10,20$ and $40 \mathrm{~kg}$ $\mathrm{mol}^{-1}$ (from right to left) in intermediate contrast and (b) $\mathrm{C}_{n}-\mathrm{PEO}, n=18,21,24,27,28,30$ (curves from bottom to top), data have been multiplied by constant factors: $C_{21}=3, C_{24}=10, C_{27}=30, C_{28}=60, C_{30}=150$. Data have been normalized to a constant $C$. Core-shell model fits are shown as solid lines. 
Table 3 Structural properties of the micelles obtained by core-shell model fits

\begin{tabular}{llrlll}
\hline Polymer & Label & $N_{\text {agg }}$ & $R_{\mathrm{m}}[\AA]$ & $R_{\mathrm{c}}[\AA]$ & $D[\AA]$ \\
\hline $\mathrm{C}_{18}$-PEO5 & hh & 28 & 78 & 15 & 63 \\
& dd & 30 & 79 & 16 & 63 \\
$\mathrm{C}_{21}$-PEO5 & hh & 45 & 87 & 19 & 68 \\
& hd & 53 & 89 & 20 & 69 \\
$\mathrm{C}_{24}$-PEO5 & hh & 75 & 103 & 23 & 80 \\
& hd & 80 & 100 & 24 & 76 \\
$\mathrm{C}_{27}$-PEO5 & hh & 96 & 109 & 26 & 83 \\
& hd & 122 & 108 & 27 & 81 \\
$\mathrm{C}_{27}$-PEO10 & hh & 39 & 142 & 18 & 124 \\
& hd & 41 & 147 & 18 & 129 \\
$\mathrm{C}_{27}$-PEO20 & hh & 41 & 208 & 18 & 190 \\
& hd & 38 & 204 & 18 & 186 \\
$\mathrm{C}_{27}$-PEO40 & hh & 30 & 282 & 16 & 266 \\
& hd & 23 & 268 & 15 & 253 \\
$\mathrm{C}_{28}$-PEO5 & hh & 95 & 110 & 26 & 84 \\
& hd & 107 & 112 & 27 & 85 \\
$\mathrm{C}_{30}$-PEO5 & hh & 125 & 111 & 30 & 81 \\
& hd & 120 & 101 & 29 & 72 \\
& & & & &
\end{tabular}

that for the studied low concentrations such a small value of $A_{2}$ has basically no effect on the results. The important fit parameters are summarized in Table 3 including $R_{\mathrm{c}}$ and $D$ calculated by $R_{\mathrm{m}}-R_{\mathrm{c}}$. The fits further yield characteristic length scales ("blob") of the corona between $40 \AA$ and $80 \AA$. These values depend strongly on the data corrections at high $Q$ where the internal shell structure is essentially determined and therefore, vary rather unsystematically with $n$. Since the "blobscattering" has only a minor effect on the global micellar parameters this is not further discussed here. Best fits were obtained by using $\sigma_{\text {int }}=5 \AA$ as a value for the core-shell smearing. For data analysis we have neglected any polydispersity effects since micelles were considered to be monodisperse. This assumption can be made for large interfacial tensions in such systems $\left(\approx 50 \mathrm{~m} \mathrm{Nm}^{-1}\right)$ and low cmc $(\leq 2 \times$ $\left.10^{-5} \mathrm{~mol}^{-1}\right) . .^{55}$ Additionally, as has already been shown in a previous work ${ }^{41}$ the aggregation behavior is not significantly affected by H/D isotope labeling (see Table 3). Hence, we can exclude any pronounced isotope effect.

\subsection{Effect of PEO molecular weight on micellar structure}

The SANS scattering curves shown in Fig. 1(a) demonstrate the influence of PEO molecular weight on the micellar structure for four different PEO $x$ molecular weights $x=5,10,20$ and $40 \mathrm{~kg}$ $\mathrm{mol}^{-1}$ at constant hydrocarbon chain length $(n=27)$. As can be seen the aggregation number clearly drops when the PEO molecular weight is increased from $5 \mathrm{~kg} \mathrm{~mol}^{-1}$ to $10 \mathrm{~kg} \mathrm{~mol}^{-1}$ whereas a further increase of $M_{\mathrm{PEO}}$ to $20 \mathrm{~kg} \mathrm{~mol}^{-1}$ and $40 \mathrm{~kg}$ $\mathrm{mol}^{-1}$ does not lead to a significant change in $N_{\text {agg }}$ anymore. The SANS curves additionally show a consistent shift of the Guinier plateau to smaller $Q$ as a natural consequence of increasing micellar size with the PEO molecular weight. The data were analyzed by the core-shell model following the same fit procedure as described in the previous section. Best fits are shown as solid lines in Fig. 1. The important fit parameters are included in Table 3.

The found aggregation numbers $N_{\text {agg }}$ are then plotted as a function of the number of EO repeat units, $N_{\mathrm{PEO}}$, in Fig. 2(a). As already qualitatively discussed above there is a steep decay in $N_{\text {agg }}$ from $\mathrm{C}_{27}-\mathrm{PEO} 5$ to $\mathrm{C}_{27}-\mathrm{PEO} 10$ while for the higher PEO molecular weights $N_{\text {agg }}$ depends only weakly on the PEO chain length. In the scaling theory for starlike micelles of Halperin ${ }^{3}$ the dependence of $N_{\text {agg }}$ on the corona chain length is not explicitly considered. There, the aggregation number essentially depends only on the size of the core block. Zhulina and coworkers $^{9}$ on the other hand have shown that in the limit of long chains $N_{\text {agg }}$ shows a weak logarithmic dependence, $N_{\text {agg }} \sim$ $(\ln N)^{-6 / 5}$. This dependence is depicted by the solid line in Fig. 2(a). Apparently, the data points are well represented for large $N_{\mathrm{PEO}}$ but do not agree for the initial strong drop between $\mathrm{C}_{27}$-PEO5 and $\mathrm{C}_{27}$-PEO10. On the basis of a pseudo mean-field phase approximation Nagarajan and Ganesh ${ }^{5}$ proposed that the corona block size has a stronger influence on the aggregation behavior, especially when the solvent is a very good solvent. For PEO-PPO micelles in water they numerically calculated the following empirical scaling relationship: $N_{\text {agg }} \sim N^{-0.51}$. This dependence is shown as a dashed line in Fig. 2(a). It can be seen
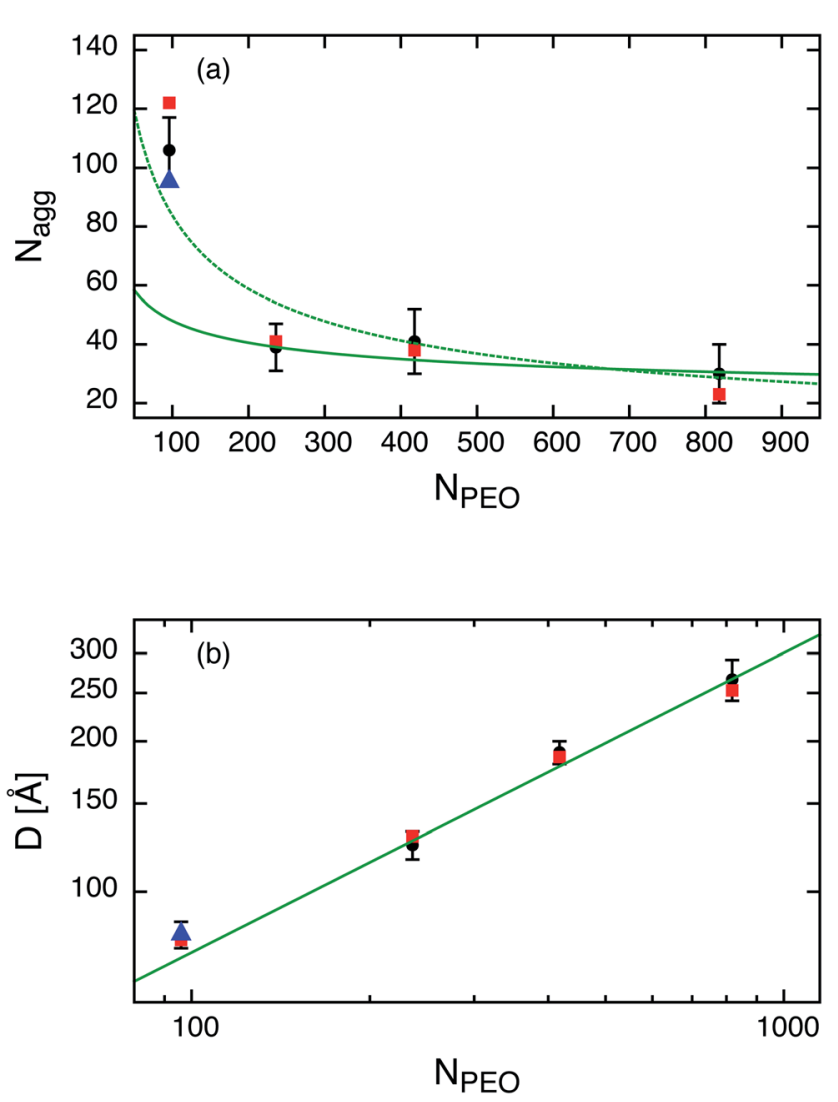

Fig. 2 (a) Aggregation number $N_{\text {agg }}$ vs. number of EO repeat units, $N_{\text {PEO }}$ : $(\boldsymbol{\bullet})$ hh labeled polymer, $(\square)$ hd labeled polymer and $(\boldsymbol{\Lambda})$ obtained under full contrast in $\mathrm{D}_{2} \mathrm{O}$. Solid line represents the scaling law of the micellar star-model, ${ }^{9}$ dashed line a semi-empirical dependence deduced by Nagarajan and Ganesh for PPO-PEO micelles. ${ }^{5}$ (b) Shell thickness $D$ vs. $N_{\text {PEO }}$ in log-log representation. Solid line represents a slope of $3 / 5$. 
that the experimental trend is well reproduced by this relationship including the data point for $\mathrm{C}_{27}-\mathrm{PEO} 5$ micelles in intermediate contrast and under full contrast measured in $\mathrm{D}_{2} \mathrm{O}$ (see triangle Fig. 2(a)). The observed decrease of $N_{\text {agg }}$ can be qualitatively explained by a change of the balance between entropic and enthalpic contributions with the growing PEO block. ${ }^{56}$ This should lead to a larger steric hindrance of the head-groups on the hydrophobic core surface such that the system is shifted to a new equilibrium structure with smaller aggregation numbers. Furthermore, Fig. 2(b) depicts the micellar shell thickness $D$ as a function of $N_{\text {PEO }}$. We see that the experimental data are in excellent agreement with the theoretical prediction $D \sim N^{3 / 5}$ for star-like micelles ${ }^{3}$ which is shown by the straight line. Thus, the aggregation behavior at constant $n$ alkyl chain length is determined by the polymer character of the hydrophilic PEO block.

\subsection{Effect of hydrocarbon chain length}

The $\mathrm{C}_{18}$-PEO5 micelles were compared to micelles formed by a commercial material Brij700 of similar chemical composition. Sommer et $a l .{ }^{31}$ have extensively studied the structure of Brij700 by using SANS and SAXS. They found a core radius of $R_{\mathrm{c}}=15-17 \AA$ and an aggregation number of $N_{\text {agg }}=30$ in very good agreement with the present results. We take this now as a starting point to systematically discuss the effect of the $n$-alkyl chain length at a constant PEO molecular weight of $5 \mathrm{~kg} \mathrm{~mol}{ }^{-1}$.

The scattering data presented in Fig. 1(b) for the different hh $\mathrm{C}_{n}$-PEO5 micellar solutions in $\mathrm{D}_{2} \mathrm{O}$ show that the intensity increases with $n$, directly demonstrating a growth of the micelles in terms of an increasing $N_{\text {agg }}$ accompanied by an increasing core size. Although the PEO molecular weight stays constant the shell thickness $D$ increases slightly (see Table 3). This might be due to a slightly higher PEO density near the core surface leading to a stronger chain stretching of the PEO block. It should be stressed again that the interfacial tension, $\gamma$, of the water- $n$-alkane interface is almost constant $\left(\approx 50 \mathrm{~m} \mathrm{Nm}^{-1}\right)$ within the higher members of the homologous series of $n$ alkanes. ${ }^{55}$ Thus, any effect on the micellar properties is mainly due to the increase of the hydrocarbon chain length. The aggregation number $N_{\text {agg }}$ as a function of the $n$-alkyl chain length is depicted in Fig. 3. We included recent experimental data found for poly(ethylene oxide)-mono- $n$-alkyl ethers $\mathrm{C}_{n^{-}}$ PEO. ${ }^{30,31,57,58}$ In order to exclude any effects of the hydrophilic block on the aggregation number $N_{\text {agg }}$ was scaled by $N_{\text {PEO }}{ }^{0.51}$ which was found above to be a reasonable description of the PEO length on $N_{\text {agg }}$. We observe that $N_{\text {agg }}$ systematically increases with increasing $n$. The system almost reveals the characteristic $n^{2}$ dependence for the aggregation number that is denoted by the solid line in Fig. 3. The deviation from this behavior is larger for the $\mathrm{C}_{18}-\mathrm{PEO} 5$ and $\mathrm{C}_{21}-\mathrm{PEO} 5$ but still in good agreement with the $n^{2}$ power law. The $n^{2}$ scaling can then be associated by either assuming a simple geometrical model as for low-molecular weight surfactants that inherently give the $n^{2}$ scaling law $^{\mathbf{2 6}}$ or the super strong segregation regime as outlined in the theoretical section. Given the hybrid nature of our

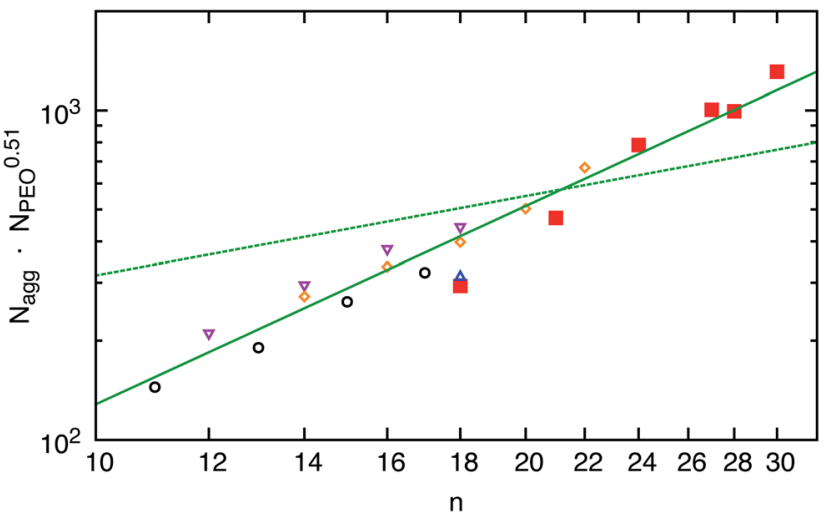

Fig. 3 Aggregation number $N_{\text {agg }}$ as a function of $n:(\square) C_{n}-P E O 5,(0)$ $\mathrm{C}_{n} \mathrm{E}_{40},{ }^{57}(\triangle) \mathrm{Brij} \mathrm{P00},{ }^{31}(\nabla) \mathrm{C}_{n}-\mathrm{PEO} 5,{ }^{58}$ and $(\diamond) \mathrm{C}_{n}-\mathrm{PEO} 5{ }^{30}$ The solid line has a slope of 2 and the dashed line of $4 / 5$ expected form the starmodel.

system, it is tempting to speculate that this behavior reflects the surfactant properties of the hydrocarbon core. This is furthermore supported by the fact that the area $a$ per molecule on the core surface does not vary substantially with $n$. Here we find a mean value of about $(93 \pm 6) \AA^{2}$ which suggest that $N_{\text {agg }} a=$ $4 \pi R_{\mathrm{c}}{ }^{2}$ is similar to what is found for surfactant micelles. ${ }^{46}$ Thus, the $n$-alkyl chain linearly increases with $n$ and if we allow a homogeneous compact core, ${ }^{59}$ it follows that $N_{\text {agg }}$ scales with $n^{2}$. A comparison of $R_{\mathrm{c}}$ with the maximal possible length, $l_{\max }$, of an alkane chain $1.53+1.265(n-1)[\AA]^{59}$ shows that the experimental values found for the micelles in the core are systematically smaller. For example for $\mathrm{C}_{24}$ we find $R_{\mathrm{c}}=23 \AA$ whereas $l_{\text {max }}=31$ A. Apparently, the alkyl chains do not assume a fully extended all-trans configuration. This was also observed by Sommer et al. ${ }^{31}$ on Brij700 where $R_{\mathrm{c}}$ is reduced by a factor of about 0.75 for $\mathrm{C}_{18}$. This corresponds to a more flexible conformation in the bulk state i.e. the all-trans configuration is perturbed due to kinks along the backbone. According to Tanford the average chain length of a more flexible $n$-alkyl chain is given by $l_{\text {flex }}=1.53+0.925(n-1)[\AA] .{ }^{59}$ The latter analytical expression nicely agrees with the experimental data e.g. $l_{\text {flex }}\left[\mathrm{C}_{24}\right]=23 \AA$. Thus, the conformation of the alkyl chains can be considered as more flexible which coincides with the assumption that the spherical core has a homogeneous density profile. It should be mentioned that long alkyl chains might crystallize ${ }^{60,61}$ partly resulting in a non-spherical core domain. Crystalline micellar cores together with the high interfacial tension and a temperature insensitive aggregation number would support micelles in the super-strong segregation limit (SSSL) where the micellar coronas are still spherical. Since the scattering data are an average over an ensemble of micelles, any deviation of the micellar core from spherical geometry is not easy to deduce directly. The rather broad core-corona interface $\left(\sigma_{\text {int }} \approx 5 \AA\right)$ might be an indication for a more elliptical shape of the core. Since, our scattering data cannot capture this possibility, it has not been considered here. But there is evidence of a phase transition, differential scanning calorimetry, density measurements and SAXS data suggest that $n$-alkyl micellar cores are partly crystalline at low enough temperatures. The discussion of 


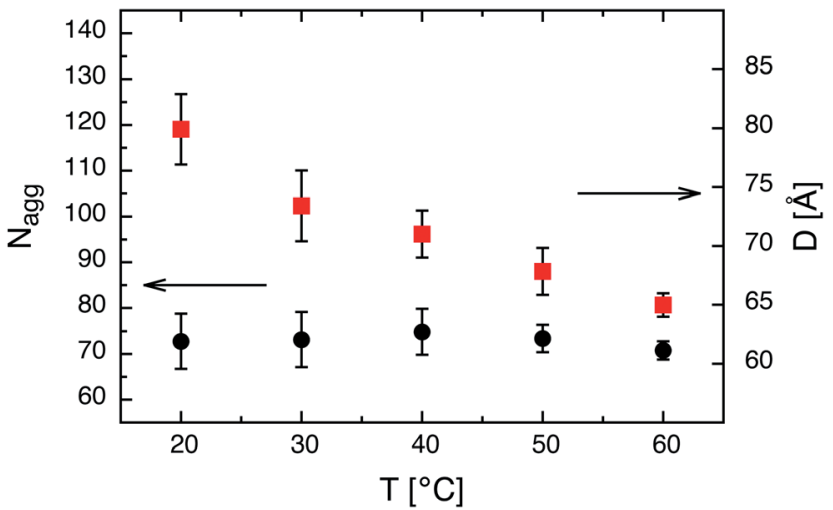

Fig. 4 Aggregation number $N_{\text {agg }}$ and corona thickness $D$ as a function of temperature $T$ for $C_{24}-$ PEO5 micelles: $(\bullet) N_{\text {agg }}$ and $(\square) D$.

these results goes beyond the scope of the present work and will be shown in an upcoming publication.

We note that for amphiphilic micelles the same scaling law is theoretically predicted by the $\mathrm{SSSL}^{8}$ and experimentally found by Förster et $a .^{13}$ A comparison of $N_{\text {agg }}$ to the theoretically predicted scaling laws for star-like block copolymer micelles ${ }^{3}$ reveals no agreement with our data. The expected power laws for star-like micelles $N_{\text {agg }} \sim N^{4 / 5}$ are too weak and clearly not represented by our data (see dashed line in Fig. 3). In addition, the effect of temperature on the micelles in thermal equilibrium was studied for $\mathrm{C}_{24}$-PEO5 and $\mathrm{C}_{30}$-PEO5 micelles in a temperature range between $20^{\circ} \mathrm{C}$ and $60^{\circ} \mathrm{C}$. This is depicted in Fig. 4 which shows the effect of temperature on the aggregation number $N_{\text {agg }}$ and the corona thickness $D$ for $\mathrm{C}_{24}$-PEO5 micelles in water. We find that the aggregation number is within the experimental uncertainty independent of temperature which might be explained by the fact that the interfacial tension is almost temperature insensitive. ${ }^{33}$ This result coincides with recent findings by Sommer et al. ${ }^{62}$ for Brij700 in water. Moreover, there is the trend of decreasing micellar size. Since $N_{\text {agg }}$ does not change with temperature this shrinkage is associated with variations in the PEO interactions in water. It is a wellestablished fact that PEO exhibits a large number of conformations strongly depending on temperature. ${ }^{63,64}$ As the temperature increases the hydration becomes less effective which leads to a conformational change i.e. a more coiled conformation of PEO. The discussion of the temperature effect follows a model that explains the existence of a lower critical solution temperature (LCST) in PEO-water systems. Thus, the conformation change is not the only reason for the PEO shrinkage but rather the induced decrease of solvent quality. The experimental results however are in qualitive agreement with recent computer simulations. ${ }^{20}$

\section{Concluding remarks}

Poly(ethylene oxide) mono $n$-alkyl ether diblock copolymers, $\mathrm{C}_{n}-\mathrm{PEO} x$, with $n=18,21,2427,28,30$ and $x=5,10,20,40 \mathrm{~kg}$ $\mathrm{mol}^{-1}$ in aqueous solution form spherical aggregates as determined by SANS. Details of the micellar structure were extracted by applying a sophisticated core-shell model including as main ingredients a diffuse star density profile for the corona, $\phi(r) \sim$ $r^{-4 / 3}$, and a constant density profile for the core region. By this, excellent description of all SANS data was feasible providing accurate structural parameters of the micelles as a function of $n$ and $x$. This allows a systematic discussion with respect to current thermodynamic models.

In particular, we experimentally verified the predicted effects of the hydrophobic and hydrophilic chain length on the aggregation behavior. As demonstrated the aggregation number of the formed micelles increases quadratically with the length of the $n$-alkyl chain. By changing the molecular weight of the PEO block the aggregation number consistently changes as predicted by the empiric power-law numerically obtained by Nagarajan and Ganesh for PPO-PEO. Moreover, the core size $R_{\mathrm{c}}$ is in accordance with an expression for the chain length of a flexible $n$-alkyl chain which coincides with the Tanfords model for a liquid-like hydrocarbon chain i.e. hydrocarbon chain having kinks along the chain. This interpretation is also related to simple geometrical constraints on the chain packing as known from low-molecular weight surfactant micelles. However, both the SSSL theory for amphiphilic block copolymers and the surfactant theory predict that the aggregation number follows a quadratic dependence on the hydrophobic block. Considering the hydrophobic $n$-alkyl block the system is still a surfactant but with the rather long PEO block a polymer character is imposed on the system. The conclusion however is that the chemical hybrid character of $\mathrm{C}_{n}-\mathrm{PEO}$ between a surfactant molecule and a block copolymer is also reflected in the equilibrium micellar properties. Thus, we consider $\mathrm{C}_{n}{ }^{-}$ PEOx polymers as a hybrid material which might close the missing gap to micelles prepared from low-molecular weight non-ionic $\mathrm{C}_{n} \mathrm{E}_{m}$ surfactants carrying only short $n$-alkyl chains and EO head-groups, respectively.

Finally, we point out that the detailed characterization of the size and shape of $\mathrm{C}_{n}-\mathrm{PEO} x$ micelles serves as an important prerequisite to study the equilibrium chain exchange kinetics of these micellar entities. Details of this study will be presented in a forthcoming paper.

\section{Acknowledgements}

R.L. greatly acknowledges grants from the Norwegian Research Council, under the SYNKNOYT program (218411 and 228573).

\section{References}

1 J. Noolandi and K. M. Hong, Macromolecules, 1983, 16, 14431448.

2 L. Leibler, H. Orland and J. C. Wheeler, J. Chem. Phys., 1983, 79, 3550-3557.

3 A. Halperin, Macromolecules, 1987, 20, 2943-2946.

4 T. Birshtein and E. Zhulina, Polymer, 1989, 30, 170-177.

5 R. Nagarajan and K. Ganesh, J. Chem. Phys., 1989, 90, 5843.

6 D. Izzo and C. M. Marques, Macromolecules, 1993, 26, 71897194.

7 Z. Gao and A. Eisenberg, Macromolecules, 1993, 26, 73537360 . 
8 A. N. Semenov, I. A. Nyrkova and A. R. Khokhlov, Macromolecules, 1995, 28, 7491-7500.

9 E. B. Zhulina, M. Adam, I. LaRue, S. S. Sheiko and M. Rubinstein, Macromolecules, 2005, 38, 5330-5351.

10 M. Wilhelm, C. L. Zhao, Y. Wang, R. Xu, M. A. Winnik, J. L. Mura, G. Riess and M. D. Croucher, Macromolecules, 1991, 24, 1033-1040.

11 J. R. Quintana, M. Villacampa, M. Munoz, A. Andrio and I. A. Katime, Macromolecules, 1992, 25, 3125-3128.

12 J. R. Quintana, M. Villacampa, A. Andrio, M. Munoz and I. A. Katime, Macromolecules, 1992, 25, 3129-3136.

13 S. Förster, M. Zisenis, E. Wenz and M. Antonietti, J. Chem. Phys., 1996, 104, 9956-9970.

14 A. Jada, G. Hurtrez, B. Siffert and G. Riess, Macromol. Chem. Phys., 1996, 197, 3697-3710.

15 H. Iatrou, L. Willner, N. Hadjichristidis, A. Halperin and D. Richter, Macromolecules, 1996, 29, 581-591.

16 P. Hickl, M. Ballauff and A. Jada, Macromolecules, 1996, 29, 4006-4014.

17 J. Bang, S. Jain, Z. Li, T. P. Lodge, J. S. Pedersen, E. Kesselman and Y. Talmon, Macromolecules, 2006, 39, 5583.

18 K. Binder and M. Müller, Curr. Opin. Colloid Interface Sci., 2000, 5, 314-322.

19 A. Milchev, A. Bhattacharya and K. Binder, Macromolecules, 2001, 34, 1881-1893.

20 S. Hezaveh, S. Samanta, G. Milano and D. Roccatano, J. Chem. Phys., 2012, 136, 124901.

21 A. Halperin, M. Tirrell and T. P. Lodge, Adv. Polym. Sci., 1992, 100, 31-71.

22 P. Alexandridis and B. Lindman, Amphiphilic Block Copolymers: Self-Assembly and Applications, Elsevier Science, 2000.

23 C. Svaneborg and J. S. Pedersen, Phys. Rev. E: Stat., Nonlinear, Soft Matter Phys., 2001, 64, 010802.

24 G. Riess, Prog. Polym. Sci., 2003, 28, 1107-1170.

25 I. Hamley, Block Copolymers in Solution: Fundamentals and Applications, John Wiley \& Sons, 2005.

26 R. Nagarajan and E. Ruckenstein, Langmuir, 1991, 7, 29342969.

27 A. Poppe, L. Willner, J. Allgaier, J. Stellbrink and D. Richter, Macromolecules, 1997, 30, 7462-7471.

28 J. S. Pedersen, C. Svaneborg, K. Almdal, I. W. Hamley and R. N. Young, Macromolecules, 2003, 36, 416-433.

29 S. Manet, A. Lecchi, M. Impéror-Clerc, V. Zholobenko, D. Durand, C. L. P. Oliveira, J. S. Pedersen, I. Grillo, F. Meneau and C. Rochas, J. Phys. Chem. B, 2011, 115, 11318-11329.

30 F. Renou, T. Nicolai, E. Nicol and L. Benyahia, Langmuir, 2009, 25, 515-521.

31 C. Sommer, J. S. Pedersen and V. M. Garamus, Langmuir, 2005, 21, 2137-2149.

32 L. Willner, A. Poppe, J. Allgaier, M. Monkenbusch, P. Lindner and D. Richter, EPL, 2000, 51, 628.

33 R. Lund, L. Willner, J. Stellbrink, A. Radulescu and D. Richter, Macromolecules, 2004, 37, 9984-9993.

34 H. Kaya, L. Willner, J. Allgaier, J. Stellbrink and D. Richter, Appl. Phys. A: Mater. Sci. Process., 2002, 74, 499-501.
35 G. V. Jensen, Q. Shi, G. R. Deen, K. Almdal and J. S. Pedersen, Macromolecules, 2012, 45, 430-440.

36 S. Förster, B. Berton, H.-P. Hentze, E. Krämer, M. Antonietti and P. Lindner, Macromolecules, 2001, 34, 4610-4623.

37 Y. Deng, R. Young, A. Ryan, J. Fairclough, A. Norman and R. Tack, Polymer, 2002, 43, 7155-7160.

38 S. Jain, M. H. E. Dyrdahl, X. Gong, L. E. Scriven and F. S. Bates, Macromolecules, 2008, 41, 3305-3316.

39 K. Mortensen, W. Brown, K. Almdal, E. Alami and A. Jada, Langmuir, 1997, 13, 3635-3645.

$40 \mathrm{H}$. Yu, A. Natansohn, M. A. Singh and I. Torriani, Macromolecules, 2001, 34, 1258-1266.

41 T. Zinn, L. Willner, R. Lund, V. Pipich and D. Richter, Soft Matter, 2012, 8, 623-626.

42 R. Lund, L. Willner and D. Richter, Adv. Polym. Sci., 2013, 51158.

43 L. Leibler, Macromolecules, 1980, 13, 1602-1617.

44 A. N. Semenov, JETP Lett., 1985, 88, 733-742.

45 I. A. Nyrkova, A. R. Khokhlov and M. Doi, Macromolecules, 1993, 26, 3601-3610.

46 J. N. Israelachvili, D. J. Mitchell and B. W. Ninham, J. Chem. Soc., Faraday Trans. 2, 1976, 72, 1525-1568.

47 R. C. Wilhoit, K. N. Marsh, X. Hong, N. Gadalla and M. Frenkel, in Landolt-Börnstein - Group IV Physical Chemistry, 1996.

48 A. Radulescu, V. Pipich, H. Frielinghaus and M. S. Appavou, J. Phys.: Conf. Ser., 2012, 351, 012026.

49 J. S. Pedersen and C. Svaneborg, Curr. Opin. Colloid Interface Sci., 2002, 7, 158-166.

50 R. Lund, V. Pipich, L. Willner, A. Radulescu, J. Colmenero and D. Richter, Soft Matter, 2011, 7, 1491-1500.

51 G. Beaucage, J. Appl. Crystallogr., 1996, 29, 134-146.

52 M. Daoud and J. Cotton, J. Phys., 1982, 43, 531-538.

53 J. S. Pedersen, J. Phys. IV, 1993, 03, 491-498.

54 J. G. Barker and J. S. Pedersen, J. Appl. Crystallogr., 1995, 28, 105-114.

55 M. Ameri, D. Attwood, J. H. Collett and C. Booth, J. Chem. Soc., Faraday Trans., 1997, 93, 2545-2551.

56 M. R. Bohmer and L. K. Koopal, Langmuir, 1990, 6, 14781484.

57 C. Booth, D. Attwood and C. Price, Phys. Chem. Chem. Phys., 2006, 8, 3612-3622.

58 F. Lafléche, D. Durand and T. Nicolai, Macromolecules, 2003, 36, 1331-1340.

59 C. Tanford, The Hydrophobic Effect: Formation of Micelles and Biological Membranes, John Wiley \& Sons, 1980.

60 P. R. Knowles, R. J. Barlow, F. Heatley, C. Booth and C. Price, Macromol. Chem. Phys., 1994, 195, 2547-2558.

61 R. G. Alamo, L. Mandelkern, G. M. Stack, C. Krohnke and G. Wegner, Macromolecules, 1994, 27, 147-156.

62 C. Sommer, J. S. Pedersen and P. Stein, J. Phys. Chem. B, 2004, 108, 6242-6249.

63 R. Kjellander and E. Florin, J. Chem. Soc., Faraday Trans. 1, 1981, 77, 2053-2077.

64 M. J. Hey, S. M. Ilett and G. Davidson, J. Chem. Soc., Faraday Trans., 1995, 91, 3897-3900. 